\title{
La negociación colectiva de empresa transnacional en Europa: dinámicas históricas, formas y retos
}

\author{
Udo REHFELDT \\ Investigador. Institut de Recherche Economique et Sociale \\ udo.rehfeldt@ires-fr.org
}

Recibido: $27-03-2013$

Aceptado: 16-06-2014

\begin{abstract}
RESUMEN:
La directiva sobre los comités de empresas europeos (CEE) de 1994, reclamada insistentemente por las organizaciones sindicales, fue un factor determinante para el posterior desarrollo de la negociación colectiva de empresa transnacional, pero fue también una fuente de dificultades para dichas organizaciones, ya que establecía, a nivel europeo, un nuevo canal de representación que se les escapaba en parte. Ante la ausencia de un marco jurídico para la negociación colectiva de empresa transnacional, las federaciones sindicales europeas (FSE) establecieron sus propias reglas internas para retomar el control de esta dinámica y afirmar un derecho exclusivo de negociación y de firma de acuerdos transnacionales. Sin embargo, dicha dinámica se enfrenta a la persistencia de otras prácticas de negociación, entre otras las de los CEE de empresas transnacionales (ETN) alemanas, pero también de otros países, que desean seguir con el control de dichas negociaciones.
\end{abstract}

Palabras clave: Comité de empresa europeo, sindicatos, empresas trasnacionales, federaciones sindicales europeas, dialogo social, directivas europeas.

\section{The Transnational Firm Collective Bargaining in Europe: Historical Dynamics, Ways and Challenges}

\begin{abstract}
The directive on European works councils of 1994, insistently demanded by trade unions, has been a decisive factor for the subsequent development of the transnational firm collective bargaining. But it has also been a source of problems for these organizations, as long as it established a new European channel of representation that was in part out of their control. Considering the lack of a regulatory framework for the collective bargaining in the transnational firms, the European trade union federations established their own internal rules in order to get the control back and support an exclusive right of bargaining and signing transnational agreements. Nevertheless, this dynamic faces the persistence of other bargaining practices. Among others, those developed by German and some other countries transnational firms that want to keep the control of their own bargaining practices.
\end{abstract}

Keywords: European works councils, trade unions, transnational firms, European trade union federations, social dialogue, European directives.

REFERENCIA NORMALIZADA.

Rehfeldt U. (2014). "La negociación colectiva de empresa transnacional en Europa. Dinámicas históricas, formas y retos". Cuadernos de Relaciones Laborales, Vol. 32, núm. 2, p. 385-410. 
SUMARIO: 1 . La debilidad de la negociación transnacional interconfederal y sectorial. 2. Los acuerdos de empresa transnacionales: un inventario. 3. Los orígenes de una estrategia sindical voluntarista. 4. Acuerdos pioneros mediante estrategias empresariales más abiertas. 5. La búsqueda de una legislación europea de apoyo. 6. Primer impulso de la negociación transnacional: los acuerdos constitutivos de CEE. 7. Nuevo impulso de la negociación transnacional: los acuerdos marco internacionales y europeos. 8. La nueva estrategia de las federaciones sindicales europeas y sus límites. 9. Conclusión: ¿Cuáles son las perspectivas para dar un nuevo impulso a los AME? 10. Bibliografía.

\section{Introducción}

Pese a considerables diferencias entre los sistemas de relaciones laborales en los países miembros, los legisladores europeos han querido establecer las condiciones necesarias para la emergencia de un sistema europeo de negociación colectiva. ${ }^{1}$ Dicho cuadro jurídico existe ya desde hace más de 20 años para una negociación colectiva transnacional interprofesional y sectorial; pero pese a todo, no ha conocido un gran dinamismo. En cambio, sigue sin existir un marco jurídico europeo para la negociación colectiva de empresa transnacional, que se ha desarrollado sobre una base simplemente voluntaria. La directiva sobre los comités de empresa europeos (CEE), relativa a los derechos de información y consulta de dichas instancias, ha sido la que ha impulsado indirectamente la negociación transnacional, primero en forma de acuerdos de procedimiento de puesta en marcha de los CEE -en la actualidad existen más de 1000- luego, desde finales de los años 90, en forma de acuerdos transnacionales sustantivos, con frecuencia negociados o iniciados por dichos CEE. Tras un breve análisis de los motivos de la debilidad de la negociación colectiva a nivel interconfederal y sectorial, nuestro artículo analizará la dinámica, las formas y los factores explicativos del auge de los acuerdos transnacionales de empresa, y finalmente, nos preguntaremos por la legitimidad de los actores que negocian y firman esos acuerdos.

\section{La debilidad de la negociación transnacional interconfederal y sectorial}

En 1984, el Acta Única, que enmendaba el Tratado de Roma, introdujo el término « interlocutor social europeo ». El Acta encargaba a la Comisión Europea la función de promover el desarrollo de un «diálogo social » con el fin de

${ }^{1}$ Este artículo recoge, en una aproximación ampliada, elementos de un artículo editado en francés bajo el título «L'enjeu des canaux multiples de représentation pour la négociation collective d'entreprise transnationale » in I. Daugareilh (dir.), Burdeos : Presses universitaires de Bordeaux 2013. Le dialogue social dans les instances transnationales d'entreprises européennes, 
favorecer a la larga relaciones contractuales. Debido a las reticencias de algunas organizaciones patronales, el diálogo social europeo conoció unos principios bastante complicados. Sin embargo, el 31 de octubre 1991, la Confederación Europea de Sindicatos (CES), las dos organizaciones patronales UNICE (ahora Business Europe) y el Centro Europeo de Empresas Públicas (CEEP) llegaron a un acuerdo en el que las organizaciones patronales se pronunciaban, por primera vez, en favor de una negociación colectiva a nivel europeo. Se incluyó dicho acuerdo en el Protocolo Social de Maastricht (1992) que constituye en la actualidad el « capítulo social» de los Tratados europeos. Con este texto, las organizaciones sindicales y patronales europeas han adquirido un estatus público y un fuerte poder en los procesos de legislación social europea. Desde ese momento, la Comisión debe consultarlos antes de cualquier iniciativa legislativa y debe, llegado el caso, dejarles tiempo para que negocien un acuerdo sobre el tema propuesto. Si estos consiguen ponerse de acuerdo, pueden pedir al Consejo de ministros que transforme el acuerdo en forma de directiva. Mediante dicho procedimiento, que se asemeja a una extensión, su acuerdo se impone a los países miembros que deben, por tanto, modificar su legislación. El capítulo social da pie también a los interlocutores sociales europeos para negociar acuerdos « voluntarios » que pueden ponerse en marcha en el plano nacional « según los procedimientos y las prácticas propios de los interlocutores sociales y de los Estados miembros ». Estos acuerdos europeos se pueden negociar tanto a nivel interprofesional como a nivel sectorial, en el marco de los « comités de diálogo social sectoriales » instaurados a partir de 1999 con la autorización de la Comisión.

Pese a ese marco legislativo favorable, los progresos materiales de la negociación colectiva europea fueron escasos (Pochet, 2005 y 2006, Degryse et al., 2011; European Commission 2012). A nivel interconfederal, se firmaron solo ocho acuerdos europeos en veinte años. Cuatro se firmaron tras consulta a la Comisión, y puestos en marcha mediante directiva. Se trata de los acuerdos sobre el permiso parental (1995, revisado en 2009), el tiempo parcial (1997) y los contratos por tiempo determinado (1999). A estos se añaden cuatro acuerdos "voluntarios » sobre el teletrabajo (2002), el estrés en el trabajo (2004), la violencia y el acoso en el trabajo (2006) y/o el mercado de trabajo inclusivo (2010).

El resultado es un poco mejor a nivel de ramas, sobre todo en aquellas en las que existen esbozos de política industrial a nivel europeo. Pero en este caso también, solo seis acuerdos se pusieron en marcha mediante una directiva: sobre el tiempo de trabajo de los marineros (1998), sobre el tiempo de trabajo en los ferrocarriles (1998), sobre el tiempo de trabajo en la aviación civil (2000), sobre las condiciones de trabajo de los trabajadores móviles en los servicios transfronterizos de los ferrocarriles (2004), sobre el convenio del transporte marítimo (2008) y sobre las lesiones en el sector hospitalario (2009). Debemos añadir un acuerdo « multisectorial» sobre el polvo de sílice cristalina (2006). Un primer inventario de los acuerdos sectoriales firmados entre 1997 y 2004 (Pochet 2005) dio lugar a un intento de categorización de 182 acuerdos en sentido amplio, denominados «documentos conjuntos», y las clases en 6 categorías: acuerdos obligados, 
recomendaciones, (códigos de conductas, etc.) con procedimiento de seguimiento, declaraciones, herramientas (de formación y de acción), reglamentos internos, posturas comunes (destinadas a las instituciones europeas). Esta última categoría concierne a casi la mitad de los acuerdos. Existen menos acuerdos obligados, el inventario solo contaba con cinco en aquel entonces.

Ninguno de esos acuerdos se refiere a cuestiones sociales como consecuencia de la exclusión del tema de los salarios de las competencias legislativas comunitarias que los interlocutores sociales tampoco quisieron rebasar con negociaciones autónomas, cuando las habían iniciado en otros campos. Por otro lado, las organizaciones sindicales definieron, en algunas ramas como la metalurgia, criterios apuntando a una forma de coordinación de las reivindicaciones y negociaciones salariales en los Estados miembros, con resultados bastante escasos (Dufresne, 2011).

Podemos encontrar una serie de motivos que justifican esa debilidad. En primer lugar, algunas organizaciones de empleadores se oponían a cualquier forma de negociación colectiva a nivel europeo. Quisieron limitar el diálogo europeo a negociaciones sobre temas no controvertidos, denominados "soft", evitando negociar temas más conflictivos. Lo mismo ocurrió a nivel sectorial; algunas organizaciones patronales tardaron en constituirse en interlocutores sociales con poder de negociación en un comité de diálogo social sectorial. Por otro lado, para vencer esos límites, se necesitaba una fuerte voluntad política por parte de la Comisión que amenazaba con iniciativas legislativas, con el fin de incitar a los interlocutores sociales a que negociasen "bajo el manto de la ley". Desde hace unos diez años, lamentamos cada vez más esa falta de voluntad política.

\section{Los acuerdos de empresa transnacionales: un inventario}

Pese a la ausencia de un encuadramiento jurídico, la negociación transnacional a nivel de empresas se realizó sobre una base voluntaria. La directiva europea de 1994 sobre los CEE desempeñó un papel indirecto en esa dinámica. Tras promover una forma particular de negociación transnacional, referente al establecimiento de los CEE, ejerció una fuerte influencia en el desarrollo de la negociación de empresa colectiva transnacional en un sentido próximo a los objetivos iniciales de las Federaciones Sindicales Internacionales (FSI). Tal y como veremos más adelante (en la parte 7), fue cinco años después de la adopción de la directiva cuando se multiplicaron los acuerdos de empresa transnacionales a nivel mundial y también a nivel europeo (da Costa et al. 2010). Si excluimos los 1200 acuerdos de establecimiento de CEE contabilizados por el Instituto Sindical Europeo (Jagodziński 2012), una base de datos de la Comisión identificó hasta julio de 2013 unos 230 acuerdos transnacionales, firmados en su mayoría en multinacionales europeas (Commissión Européenne 2013). Un análisis de los acuerdos hasta el final de 2011 (da Costa y Rehfeldt 2012a) arroja el siguiente reparto. Más o menos la mitad son acuerdos marcos internacionales (AMI) firmados por FSI. Tienen un alcance geográfico mundial y abordan sobre todo los derechos fundamentales en el 
trabajo. Por analogía, la otra mitad puede ser considerada como acuerdos marcos europeos (AME). ${ }^{2}$ No tienen sino un alcance regional (europeo) y abordan temas más variados; en primer lugar, restructuraciones (en un sentido amplio que abarca la anticipación del cambio, cf. da Costa y Rehfeldt 2012b), pero también otros temas como la responsabilidad social de las empresas o la salud-seguridad. Algunos temas son temas "softs", o sea, bastante consensuales.

En algunos casos, son las empresas transnacionales las que asumen la negociación, aunque no es el caso de todos los acuerdos. Por ejemplo, algunos acuerdos de restructuraciones se negociaron en un contexto muy conflictivo y con jornadas de acción transnacionales previas. Los AMI y los AME tienen características y dinámicas muy variadas, pero poseen también rasgos comunes. Uno de estos rasgos comunes es la dimensión europea de sus acuerdos. En efecto, la mayoría se firmó en empresas transnacionales (ETN) europeas, para ser más precisos, unas ETN cuya sede se encuentra en el continente europeo. Los CEE desempeñaron un papel importante como firmantes y también en el seguimiento de los acuerdos.

Las ETN francesas firmaron el mayor número de AMI (27 hasta 2011); las seguían las ETN alemanas (21), y luego las suecas (10) y las noruegas (8). ${ }^{3}$ Por el

\footnotetext{
${ }^{2}$ No siempre es fácil hacer la distinción entre un acuerdo firmado por un CEE y una simple acta de sesión firmada por un miembro de un CEE. Esta dificultad refleja muy bien la delgada frontera que separa, a veces, una negociación de un procedimiento de consulta. Muchos acuerdos negociados por unos CEE tienen un carácter informal y no se hacen públicos. Debemos recordar aquí que, aunque la Comisión Europea ha puesto en marcha un banco de datos sobre los acuerdos de empresa transnacionales, no existe ninguna obligación de tener un depósito legal de dichos acuerdos. Un estudio alemán sobre el sector metalúrgico (Müller et al. 2013) estima en una tercera parte los ACE informales no hechos públicos. Por este motivo, debemos considerar que el número de AME inventariados es mínimo - al contrario de los AMI cuyas FSI firmantes realizan un inventario preciso, sistemático y puesto al día.

${ }^{3}$ Los acuerdos transnacionales de las ETN españolas constituyen un caso particular. En primer lugar, las ETN con sede en España son generalmente de un tamaño modesto. A pocas les afectan las obligaciones de la directiva europea sobre los CEE. Además, tienen escasa tendencia a crear un CEE, probablemente debido a la débil presencia sindical en España y de una patronal muy etnocéntrica (White y Vallejo 2004; Köhler et Gonzalez Begega 2007). Antes de 2004, habían creado un CEE seis, una de ellas era una empresa americana que trasladó posteriormente su sede a Hungría. Según la base de datos del Instituto Sindical Europeo (ETUI), a finales de 2013 eran nueve. Algunas grandes empresas españolas del sector de telecomunicaciones y de energía han emprendido, después de su privatización, un proceso de internacionalización, pero más en dirección a América Latina que hacia Europa. De ello resulta que firman más AMI que AME. La primera ETN española que ha firmado un AMI es la Telefónica en 2001, seguido de Endesa en 2002, y de Inditex (Zara) con dos acuerdos firmados en 2007 y 2009. Es en 2012-2013 cuando su cifra ha aumentado
} 
número de empresas firmantes, las empresas alemanas predominan; pues muchas empresas francesas firmaron más de un AMI - Danone firmó, ella sola, una tercera parte. Por primera vez, aparecen en 2008 unas ETN no europeas firmantes de AMI : existen ahora 5 originarias de Brasil, 4 de Estados Unidos, 3 de África del Sur, 2 de Japón, 2 de Indonesia y una de Australia, Nueva Zelanda, Canadá y Malasia respectivamente. En 2008, una ETN británica firmó por primera vez un AMI.

El predominio de las empresas francesas es aún más evidente en el caso de los AME. Cerraron casi la mitad del conjunto firmados hasta 2011, es decir, 47 de 111. Al igual que en el caso de los AMI, figura un gran número de empresas antiguamente nacionalizadas entre las empresas francesas firmantes de AME. Hay que constatar que el segundo puesto en el caso de los AME corresponde a las empresas cuya sede está en Estados Unidos, con 15 acuerdos firmados hasta 2011, cuando dichas empresas firmaron pocos $\mathrm{AMI} .{ }^{4}$ Conviene señalar que la sede europea de dichas empresas está en Alemania. Las empresas alemanas propiamente dichas ocupan sólo la tercera posición en materia de firmas de ACE, con 14 acuerdos firmados en 2011. Sobre el total de empresas que han firmado estos acuerdos 21 son francesas, seguidas de 12 alemanas.

bruscamente, con una renovación del acuerdo de Inditex en 2012 y cuatro nuevos acuerdos (OHL, Ferrovial, FCC Construcción en 2012 y Melia en 2013), elevando el número de total de ACI firmados a nueve. Desde el punto de vista del número de AMI firmados, las ETN españolas ocupan en la actualidad el quinto lugar, después de las ETN noruegas. En el momento de su firma ninguna de estas empresas había puesto todavía en marcha un CEE. Telefónica ha puesto en marcha un CEE en 2013, pero sólo para los establecimientos europeos de su filial británica Telefonía Europe, con exclusión de los establecimientos españoles de Telefónica y de sus demás filiales europeas. Según el banco de datos de la Comisión Europea sólo una ETN española ha firmado un AME: una "declaración conjunta" sobre la igualdad entre hombres y mujeres firmada por el CEE y la dirección del Banco de Santander en 2008. Con ocasión de un seminario europeo (Fundación $1^{\circ}$ de Mayo, 2014) se supo que este banco a firmado después otros dos AME, uno en 2009 y otro en 2011. He ahí otro ejemplo de AME « informales » no repertoriados que se han señalado más arriba en la nota 2.

${ }^{4}$ Debido a la cultura propia de las relaciones laborales en Estados Unidos, a las ETN norteamericanas no les suele gustar reconocer a las FSI como interlocutores para la negociación colectiva transnacional; tampoco les gusta reconocer los derechos fundamentales a la sindicalización y a la negociación colectiva. 


\section{Los orígenes de una estrategia sindical voluntarista}

Los primeros intentos para instaurar una negociación colectiva a nivel de empresas transnacionales se remontan a finales de los años 1960 y principios de los años 1970 (Rehfeldt 1993; Da Costa y Rehfeldt 2008). Unas Secretarías Profesionales Internacionales (SPI), llamadas en la actualidad Federaciones Sindicales Internacionales (FSI), sobre todo las de la metalurgia, la química y la alimentación, tres ramas especialmente afectadas por el proceso de internacionalización de las empresas, se encargaron del proyecto. Concibieron dos instrumentos para alcanzar ese objetivo: la creación de consejos mundiales de grupo y el desencadenamiento de huelgas transnacionales de solidaridad. Charles Levinson, Secretario General adjunto de la Federación Internacional de trabajadores de las industrias metalúrgicas (FITIM), y después, entre 1964 y 1985, Secretario General de la Federación Internacional de trabajadores de química (ICF), ideó una ambiciosa estrategia sindical transnacional en tres fases:

- en la primera fase, se organizaría el apoyo internacional a un sindicato que pondría en marcha un conflicto laboral en una filial de un grupo transnacional ;

- en la segunda fase, habría que coordinar negociaciones colectivas simultáneas en varias filiales de un mismo grupo en varios países ;

- en la última fase, se realizarían negociaciones integradas con la dirección del grupo transnacional, basadas en reivindicaciones definidas conjunta y previamente por los distintos sindicatos nacionales (Levinson 1974; 88-105).

Era la época del apogeo de un sindicalismo de lucha para el que la relación de fuerzas parecía muy favorable en el ámbito de las grandes empresas industriales. En algunos países como Italia o el Reino Unido, las luchas sindicales habían conseguido resultados espectaculares en materia de salarios y condiciones laborales. Levinson y los SPI pensaban que se podría trasladar dicho éxito al plano internacional.

En 1966 la FITIM consiguió poner en marcha, en algunas ETN de la industria automóvil, los primeros consejos de grupos mundiales a modo de estructura de coordinación sindical. En la década siguiente, se crearon más de 60 de estos consejos en el conjunto de los sectores (Tudyka 1986). Ese número suponía un resultado aceptable, aunque escaso comparándolo con la totalidad de las ETN consideradas. Fue más difícil obtener resultados en materia de apoyo internacional a un conflicto laboral en una ETN, elemento esencial para la fase 1 de la estrategia de Levinson. En 1972, se pudo poner en marcha la primera huelga («jornada de acción ») transnacional en una ETN, conjuntamente en las filiales italianas y británicas del grupo Dunlop-Pirelli. Sea como fuere, la ICF de Levinson no pudo inventariar dicha huelga como éxito de su organización, pues la iniciativa fue 
tomada por un comité de acción y no fue coordinada por el consejo de grupo Dunlop-Pirelli que la ICF había establecido por las mismas fechas. En Italia, fue sobre todo el sindicato CGIL, mayoritario en Pirelli, el que lideró la huelga; este sindicato no estaba afiliado aún a la ICL y se veía excluido, por tanto, del consejo de grupo (Piehl 1974; Moore 1978). Se iba a producir una situación semejante en Michelin donde un comité de coordinación europeo, creado por militantes sindicales de base en 1969, coexistía con un consejo de grupo creado por la ICF en 1971, y cuyos sindicatos comunistas se veían excluidos también. En 1975, el comité de militantes organizó también una jornada de acción europea en la que no participaron los sindicatos afiliados a la ICF, menos en el Reino Unido (Philip 1978). Pese a todo, estas jornadas de acción transnacionales no tuvieron continuación durante mucho tiempo. ${ }^{5}$

En lo referente a la fase 2 de su estrategia, Levinson dio en 1972 algunos ejemplos de éxitos de negociaciones colectivas coordinadas, especialmente en las filiales de grupos norteamericanos en Estados Unidos y Canadá (Levinson 1972; 132); sin embargo, debemos señalar el carácter particular de este ejemplo, pues los trabajadores concernidos eran, por aquellas fechas, miembros de las mismas organizaciones sindicales que abarcaban de modo simultaneo ambos países. Además, tales éxitos de negociación transnacionales no duraron, pues posteriormente, las secciones canadienses de dichas organizaciones se iban a independizar de estos sindicatos "internacionales".

El mayor fracaso de la estrategia de Levinson tiene que ver con su fase final, es decir, la negociación directa con una ETN. En efecto, ninguna dirección de ETN quería, por aquel entonces, reconocer un consejo mundial como interlocutor o firmar un acuerdo transnacional con un SPI. Habría que esperar hasta 1988 para que una empresa transnacional francesa, BSN, (en la actualidad Danone) aceptase firmar el primer « acuerdo-marco internacional » (AMI) con un SPI, en este caso, la Unión Internacional de Trabajadores de la Alimentación (UITA).

¿Cómo explicar, en un primer momento, el fracaso de esta precoz estrategia de negociación colectiva transnacional? ¿Por qué una estrategia voluntarista, que conoció algunos éxitos en el plano internacional, no funcionó a nivel transnacional? Podemos avanzar dos factores explicativos. En primer lugar, era más complicado organizar acciones de solidaridad a nivel transnacional que a nivel nacional (nivel este en el que resultan ya problemáticas entre diferentes lugares de un grupo en el mismo país). Las diferencias entre sistemas jurídicos nacionales constituyen uno de los factores restrictivos, pero los obstáculos más importantes para la coordinación

5 Hubo incluso una pérdida de memoria histórica. En efecto, en 1997, a raíz del Caso Vilvoorde, se organizó una jornada de acción transnacional en las filiales europeas de Renault; los observadores la consideraron como la "primera » huelga transnacional en Europa (Rehfeldt 1998a). 
sindical transnacional eran de tipo sociológico y organizacional. Uno de dichos obstáculos era la división sindical que prevalecía entonces a nivel internacional. Con frecuencia, los sindicatos más combativos, necesarios para el éxito de la estrategia de Levinson, y que se proponían obligar a la dirección de un grupo a reconocer un SPI como interlocutor, y para que aceptara negociar un acuerdo transnacional, no estaban afiliados a los SPI. Se trataba, sobre todo, de los sindicatos comunistas, que solían ser mayoritarios en las filiales francesas o italianas, pero también de un sindicato como la CFDT, antes de que sus federaciones se afiliasen a los SPI en los años 1970. En cuanto a los sindicatos comunistas, no pudieron afiliarse, en la mayoría de los casos, antes de los años 1990, tras su abandono de la Federación Sindical Mundial FSM, en declive tras la caída del comunismo en Europa.

\section{Acuerdos pioneros mediante estrategias empresariales más abiertas}

Al no lograr construir una relación de fuerzas favorable en las ETN, pese a la creación de consejos de grupo, las SPI acabaron siendo tributarias de la emergencia de un management más abierto al diálogo en algunas de ellas. Esa apertura no se consiguió antes de 1988 con el grupo BSN-Danone. La personalidad de su Presidente Director General, Antoine Riboud, desempeñó un papel determinante: Riboud era conocido por su compromiso social inspirado en la ética católica. Es uno de los pocos Presidentes Directores Generales de una gran empresa francesa que es miembro del Partido Socialista (Gallin, 2008). Sin embargo, Danone seguiría siendo, durante mucho tiempo, la única ETN firmante de «acuerdos marcos transnacionales » (cinco entre 1988 y 1994).

El movimiento sindical, aunque no sea más que a nivel europeo en este caso, halló condiciones parecidas de apertura del management tras la nacionalización de las grandes ETN francesas por parte del gobierno de izquierda, en Francia en 1982 (da Costa y Rehfeldt 2008). Por el lado sindical, la Federación Europea de Metalúrgicos (FEM) desempeñó en este caso un papel pionero. En el plano organizacional, la FEM se independizó de la FITIM, de la que heredó el seguimiento de las actividades de las ETN a nivel europeo. Estableció entonces grupos de trabajo en algunas de las firmas que reunían regularmente a responsables sindicales de las filiales europeas de dichas empresas. Uno de los grupos de trabajo, el del grupo francés nacionalizado Thomson Gran Público (TGP) consiguió abrir un diálogo con la dirección francesa del grupo para hablar de los problemas de empleo debidos a proyectos de restructuración en el sector de la electrónica para el gran público. El momento para el encuentro era propicio, ya que, tras la nacionalización del grupo, la dirección se había topado con una fuerte oposición sindical en Alemania, cuando decidió cerrar allí una fábrica que se dedicaba a la fabricación de televisores. Manifestó entonces la intención de establecer relaciones de mayor confianza con los sindicatos en las filiales europeas, con el fin de a borrar la mala imagen que aquella decisión dejó en ellos. El 7 de Octubre de 1985, dos acuerdos 
fueron firmados por la dirección de TGP, la FEM y unos cuantos responsables sindicales de TGP y de sus filiales en cuatro países europeos. Dio pie al nacimiento de una doble estructura de información y consulta: un «comité de unión TGPFEM » y una «comisión europea de rama ». Se creó esta última instancia para asociar también sindicatos que, por aquellas fechas, no estaban afiliados aún ni a la FEM, ni a la CES, como la CGT francesa, la CGC francesa y las Comisiones Obreras españolas. Más adelante, se crearon « comités europeos de grupo », bien mediante decisión unilateral de las direcciones, bien mediante acuerdo con federaciones sindicales europeas y/o nacionales en otros 17 grupos franceses nacionalizados. ${ }^{6} \mathrm{El}$ gobierno francés recomendaba encarecidamente las iniciativas de las ETN francesas. Así pues, en julio de 1989, el Primer Ministro Michel Rocard envió una carta a los PDG de las empresas nacionalizadas recomendándoles un comportamiento "ejemplar" en materia de diálogo social y pidiéndole entre otras cosas que establecieran unos CEE sobre una base voluntaria (Rehfeldt 1998b).

Uno de los objetivos de las direcciones de las ETN francesas consistía en hacer emerger, mediante la negociación de estos acuerdos, una identidad de empresa europea, incluso entre los asalariados y sus representantes sindicales. Por aquel entonces, se consideraba necesaria y urgente esa iniciativa, y más teniendo en cuenta que las empresas de reciente nacionalización, a semejanza de Thomson Gran Público, tenían muy mala prensa en los países vecinos que consideraban que perseguían objetivos puramente nacionales, en detrimento del empleo en las filiales extranjeras. Varios proyectos de cierre de empresas contribuyeron, con o sin razón, a reforzar esa mala fama. Una mejora del diálogo con los representantes de los asalariados en el extranjero se imponía por tanto como objetivo prioritario para el nuevo management de dichas empresas. De modo indirecto, la creación de un comité europeo de grupo tenía que contribuir también a la homogeneización y al control de la gestión social de las filiales extranjeras del grupo, y a la creación de una cultura común de relaciones sociales.

Las mismas motivaciones aparecieron también en el management de otras ETN, sobre todo alemanas. Tras el éxito del acuerdo europeo de 1985 con Thomson Gran Público, la FEM pidió en 1988 a sus organizaciones afiliadas que iniciasen

${ }^{6}$ Se trataba de Bull (1988), Saint-Gobain (1989), Pechiney (1990), RhônePoulenc (1990), Elf-Aquitaine (1989), Europipe (1991), AGF (1991), Airbus Industrie (1992), Eurocopter (1992), Saint-Gobain (1992), Renault (1993), ThomsonCSF (1993), Générale des Eaux (1993), Schneider (1993), Usinor-Sacilor (1994), Accor (1994), Crédit lyonnais (1994). En Saint-Gobain, hubo un precedente con la constitución de un comité europeo en un sector del vidrio en 1983, con una participación que se limitaba a los sindicatos afiliados a la ICF; pero no tuvo sino una existencia efímera que terminó tras dos reuniones en 1985. (Para el historial del CEE de Saint-Gobain cf. González Begega 2011). 
negociaciones con las direcciones centrales de las ETN con el fin de firmar acuerdos para la instauración de instancias europeas de información y consulta. En 1989, la IG Metall alemana recogió esta recomendación en un documento en el que aconsejaba la negociación para el establecimiento de «comités económicos europeos» (Rehfeldt 1993). El término «comité económico» se refería a la legislación alemana sobre los derechos de codeterminación de los consejos de empresa. En las empresas de más de 100 asalariados, existe un derecho de información y de consulta regular sobre asuntos económicos de una comisión particular del consejo de empresa, denominada "comité económico ${ }^{7}{ }^{7}$ Dicha particularidad terminológica es reveladora de las dificultades que tuvo IG Metall para aplicar las recomendaciones de la FEM en el contexto de la legislación alemana. Estas dificultades supusieron algunas diferencias en relación con las recomendaciones y con los primeros acuerdos europeos negociados con grupos franceses. Entre otras cosas, se refieren al modo de designación de los miembros. En el modelo dual alemán, el consejo de empresa constituye un canal de representación jurídicamente autónomo en comparación con la representación sindical que es exterior a la empresa. Aplicar el mismo modelo a la futura instancia europea, implicaba por tanto para IG Metall que los miembros alemanes de la instancia europea debían ser elegidos por el personal o sus representantes. La futura instancia debía tener derecho también a recibir la asistencia de un experto sindical externo a la empresa, aunque no de un representante de la FEM, tal y como preveía el proyecto de esta. Dicha divergencia no se solucionó antes de 2000 mediante un compromiso dentro de la FEM que preveía la designación de un coordinador FEM para cada CEE de acuerdo con la organización sindical mayoritaria en el país de origen de la ETN implicada. En la práctica, dicho coordinador suele ser un funcionario sindical originario de este país.

El primer resultado de la iniciativa de IG Metall, fue la constitución de un « consejo de empresa de grupo europeo » en Volkswagen en 1990, el primer CEE de una ETN no francesa. Formalmente, no es el sindicato sino el consejo central de empresa de la sede el que ha negociado su establecimiento. Sin embargo, en Volkswagen casi todos los consejeros de empresa elegidos eran miembros de IG Metall, lo mismo que un $90 \%$ de los asalariados Volkswagen en Alemania. En un principio, se estableció el nuevo CEE, al igual que en algunas ETN francesas, basándose en una decisión unilateral de la dirección. Aunque asumió los gastos de reunión, no fue antes de 1992 cuando reconoció formalmente la nueva instancia, mediante acuerdo firmado. Por el lado de los representantes de los asalariados,

${ }^{7}$ IG Metall abandonó después el término «comité económico europeo » por el de «consejo de empresa europeo »; fue el término que la Comisión Europea escogió finalmente en la versión alemana de su proyecto de directiva adoptado en 1994. 
dicho acuerdo fue firmado por la propia instancia europea. A diferencia de los acuerdos franceses, no se trata por tanto de un acuerdo sindical. La iniciativa de Volkswagen, que no es miembro de la federación alemana de empleadores de la metalurgia, fue al principio la única dentro de esta rama, pero el procedimiento elegido inspiró el establecimiento en 1991 de instancias europeas en dos ETN alemanas de la industria química, Bayer y Continental.

\section{La búsqueda de una legislación europea de apoyo}

A principios de los años 1990, la negociación colectiva de empresa transnacional siguió por tanto tributaria de la voluntad de apertura de un pequeño número de direcciones de ETN, en este caso de empresas nacionalizadas francesas y de algunas grandes empresas alemanas con tradición de «cooperacion social» (Sozialpartnerschaft) como Volkswagen y algunas empresas químicas. Tentativas paralelas de la FEM para firmar acuerdos similares con otras ETN, sobre todo de origen anglosajón habían sido un fracaso. Lo cual indicaba perfectamente los límites de una estrategia sindical meramente voluntarista. De hecho, el movimiento sindical se había temido muy pronto dicho límite y pidió que se instaurase una legislación de apoyo a nivel europeo. Reivindicó también dicha legislación en otras organizaciones internacionales, como las Naciones Unidas, la Organización Internacional del Trabajo (OIT) o la OCDE. Sin embargo, estas peticiones, compartidas por algunos Estados, sólo habían conseguido códigos de conducta no obligatorios como los «Principios directores dirigidos a las empresas multinacionales» de la OCDE en 1976, y en 1977, la Declaración tripartita sobre las empresas multinacionales y la política social de la OIT. ${ }^{8}$ No era más que en el plano de la Comunidad Europea donde los tratados y las instituciones supranacionales dejaban esperanza para la instauración de una verdadera regulación social en forma de legislación de apoyo para la representación y la negociación colectiva en las ETN a nivel europeo.

Un primer intento al respecto consistió en un proyecto de la Comisión Europea de un estatuto de la « sociedad anónima europea », presentado en 1970. Este

${ }^{8}$ Los Principios directores de la OCDE fueron revisados varias veces. Desde el año 2000, incluyen un procedimiento reforzado que permite cuestionar públicamente una empresa individual. La Declaración Tripartita de la OIT también fue revisada varias veces, pero sigue sin incluir sanciones. El código de la ONU nunca se adoptó. Su Secretario General, Kofi Annan, lanzó en 2000 un "Pacto mundial» (Global Compact) al que las empresas pueden adherir voluntariamente. Solo corren el riesgo de verse tachadas de la lista de adherentes si no respetan sus compromisos. 
proyecto incluía, de modo simultáneo, tres canales de « participación » de los trabajadores:

- la negociación de convenios colectivos europeos firmados directamente entre la sociedad europea y los sindicatos representados en los distintos establecimientos ;

- un comité de empresa europeo (CEE) al que se debía informar regularmente sobre la evolución de los parámetros económicos y sociales de la empresa y consultar ante cualquier toma de decisión importante ;

- la participación de representantes de los trabajadores en el consejo de vigilancia para, al menos, una tercera parte de sus miembros; cierto número de dichos representantes debía ser obligatoriamente externos a la empresa.

Aunque dicho proyecto de la Comisión intentase combinar, de modo original, los distintos modelos nacionales europeos, previendo varios canales de representación, se enfrentó a la oposición de las organizaciones patronales, y también de la mayoría de las organizaciones sindicales, sobre todo por su hostilidad al modelo alemán de codeterminación que inspiraba dos de los tres canales de representación previstos por la Comisión (Rehfeldt 2009). El proyecto de estatuto para la sociedad europea se quedó paralizado durante más de treinta años y no consiguió resultados, bajo una forma muy diferente, hasta 2001. En lo referente al encuadramiento jurídico de una negociación colectiva europea, el dossier logró, gracias al protocolo social de Maastricht de 1991, la posibilidad, para las organizaciones sindicales y patronales, de firmar acuerdos transnacionales a nivel interprofesional y sectorial, aunque su alcance fuera limitado debido al rechazo británico de transferir competencias legislativas en materia de derecho sindical y de derecho a la huelga al ámbito europeo.

La negociación colectiva, a nivel de las ETN, quedó sin encuadramiento legislativo. No fue hasta 2004 cuando la Comisión Europea anunció su intención de proponer un marco europeo "opcional" para la negociación colectiva transnacional. Pero, debido a la ausencia de consenso de los interlocutores sociales europeos sobre la necesidad de dicho marco - la CES era favorable y Business Europe opuesto - la Comisión Europea renunció de momento a presentar un proyecto y prefirió seguir con su reflexión mediante peritaje y seminarios.

En lo referente al dossier del CEE, fue objeto en 1980 de un primer proyecto de directiva, la "directiva Vredeling », que establecía un derecho de información y consulta de los representantes de los asalariados en las ETN sobre todo en caso de restructuración. En caso de proyecto de cierre total o parcial de una filial, se preveía una consulta en dos fases. La primera a nivel de la filial, donde los representantes de los asalariados disponían de un plazo de treinta días, tras recibir la información, para formular una recomendación. Si la decisión prevista "pudiese afectar directamente sus condiciones de empleo y de trabajo », la dirección debía iniciar negociaciones con ellos. Si estimaban que la información dada por la dirección 
local era insuficiente, el proyecto de directiva les daba también derecho a hablar directamente con la dirección de la sede, incluso si se hallase fuera de la Comunidad Europea. En tal caso, se podía constituir una representación de los trabajadores para el conjunto de las filiales europeas, de acuerdo con la sede. En este caso, las disposiciones para la representación de la filial se aplicarían a dicha instancia. Según su autor, el comisario socialista holandés Henk Vredeling, dicho proyecto de directiva se proponía también favorecer la negociación colectiva transnacional. Sin embargo, el Consejo de Ministros nunca lo pudo adoptar, debido a la necesidad de una votación unánime y de la amenaza del gobierno británico de Margaret Thacher de utilizar su derecho a veto.

Fue necesario un cambio de los Tratados europeos, que permitía la adopción de una directiva sobre la información-consulta por mayoría cualificada, para que el Consejo de Ministros adoptase finalmente, en septiembre de 1994, un nuevo proyecto, denominado directiva sobre los CEE. La directiva, que entró en vigor en septiembre de 1996, recoge buena parte del proyecto Vredeling, salvo la idea inicial de una negociación en caso de restructuración. No incluye ni obligación de información previa sobre un proyecto de restructuración, ni obligación de consulta de cara a conseguir un acuerdo. En cambio, es obligatoria una negociación para la constitución de una instancia de información y consulta en todas las empresas transnacionales que emplean al menos 1000 trabajadores dentro del Espacio Económico Europeo (UE más Noruega, Islandia y Liechtenstein) y al menos 150 trabajadores en más de un país miembro. A petición de al menos 100 asalariados (o sus representantes) en dos países al menos, la dirección de un grupo transnacional implicado debe entablar negociaciones con una comisión negociadora (GEN), compuesto de representantes de los asalariados del conjunto del grupo, para establecer un CEE o un procedimiento de información-consulta equivalente. Cada dirección de empresa implicada dispone de tres años, a partir de una petición hecha por un grupo de asalariados o sus representantes, para negociar el establecimiento de un CEE o de un procedimiento de información-consulta. En caso de rechazo de negociación, de desacuerdo manifiesto o tras expiración del plazo de tres años, un anexo de la directiva define "requisitos subsidiarios » para un CEE, que se aplicarán entonces automáticamente. Se supone que eso ayuda a la comisión negociadora a negociar un acuerdo que establezca reglas al menos equivalentes a las de dichos requisitos. Según el artículo 13, los grupos que han negociado ya un " acuerdo voluntario », o sea con los interlocutores que han elegido, antes de la entrada en vigor de la directiva, tienen derecho a conservar su acuerdo tal cual y de verse eximidos de una obligación de renegociación con una comisión negociadora compuesta según las reglas definidas por el artículo 6 y las leyes nacionales de transposición En caso de que semejante acuerdo se hubiera firmado por tiempo limitado, las partes firmantes tendrán derecho a prorrogarlo y a revisarlo libremente tantas veces como quisieran. 


\section{Primer impulso de la negociación transnacional: los acuerdos constitutivos de CEE}

El artículo 13 de la directiva fue el que impulsó una forma particular de negociación colectiva de empresa. En primer lugar, tenía poder de ley para los 36 acuerdos voluntarios firmados en unas ETN antes de la adopción de la directiva. Otras 350 ETN aprovecharon dicho artículo para firmar también acuerdos «voluntarios », la tercera parte en el mes anterior a la expiración del plazo de transposición de la directiva. El procedimiento de negociación era entonces totalmente libre y dejaba a las empresas una gran posibilidad de elección de los interlocutores. Pese a esta libertad, las tres cuartas partes de los acuerdos denominados art. 13 fueron firmados o cofirmados por federaciones sindicales europeas (FSE). Por lo que respecta a los acuerdos firmados según el art. 6, la directiva no da ningún papel particular a las organizaciones sindicales - que no se mencionan, además, en ninguna parte. Como mucho, la directiva autoriza a la comisión negociadora a que le asiste un experto externo ${ }^{9}$. Las FSE recomiendan encarecidamente a las comisiones negociadoras que nombren para ello un experto sindical. Una mayoría de comisiones negociadoras aplicó esta recomendación, y de ello resulta que parte de los « acuerdos artículo 6 » son cofirmados por una FSE. En 2006, el 27\% del conjunto de los 806 acuerdos CEE eran firmados o cofirmados por una FSE. Dicha tasa es mucho más elevada en el caso de los acuerdos firmados en las ETN francesas $(57 \%)$ e italianas (54\%), pero es muy inferior en las alemanas (10\%) (Kerckhofs 2006).

Debemos recordar una vez más que ese importante número de acuerdos firmados por las FSE no es sino el resultado de su propia iniciativa autónoma. En efecto, tras imponer un monopolio sindical para la participación en la negociación europea interprofesional y sectorial, mediante el protocolo social de Maastricht, en 1992, el legislador europeo instauró claramente un nuevo canal de representación mediante la directiva sobre los CEE de 1994. Sin embargo, los sindicatos no se ven totalmente excluidos de este canal de representación, pues algunas leyes nacionales de transposición establecieron un derecho sindical para el nombramiento de los miembros de la comisión negociadora y del CEE (según las prescripciones subsidiarias), siempre y cuando dichos sindicatos estén presentes en los establecimientos implicados. Dicho derecho existe en los países de canal

\footnotetext{
${ }^{9}$ No es sino la versión revisada de la directiva de 2009 que menciona por primera vez las organizaciones sindicales europeas. En efecto, se las cita como ejemplo de un experto externo de la comisión negociadora, aunque no da ningún nuevo derecho en relación con la situación preexistente. Y dato más importante, la directiva revisada obliga a las empresas a notificar a la FSE implicada un inicio de negociación con una comisión negociadora. Lo cual mejora las posibilidades sindicales de tener influencia en el curso de las negociaciones.
} 
único como el Reino Unido e Italia, y en los países de monopolio sindical de negociación colectiva como Francia. Sin embargo, solo se aplica a los representantes asalariados elegidos en los distintos establecimientos. Ya que muchos acuerdos establecen procedimientos de designación cercanos a los requisitos subsidiarios, la medida permitió una presencia sindical significativa en los CEE de las mayores ETN. En dichas ETN, los representantes elegidos por otro canal, como el del consejo de empresa en Alemania, suelen estar afiliados a un sindicato.

Mencionamos que las organizaciones sindicales desearon encarecidamente dicha legislación europea de apoyo. Aunque la directiva sobre los CEE de 1994 no correspondiera sino parcialmente a su deseo, sí apoyaron activamente el establecimiento y la acción de los CEE. Sin embargo, en lo referente a la utilización de los derechos de información y consulta, el balance de dichos comités muestra una gran variedad de experiencias (Waddington, 2012). En los acuerdos, unos procedimientos apropiados que permitieran un verdadero debate informado y una posible inflexión de los puntos de vista solían estar ausentes. Con frecuencia, las empresas se olvidan informar a los CEE de sus proyectos de restructuración, cuando es, sin embargo, la principal razón de ser de dicha legislación. La cuestión del carácter previo (« con suficiente antelación ») de la información se planteó con el cierre de la filial belga de Vilvorde del grupo Renault en 1997. Dicha cuestión centraba el debate que acabó con la revisión de la directiva en 2009 y que reforzó los derechos de información y consulta de los CEE (Jagodziński 2012). La directiva revisada recomendaba que la información debía efectuarse « en un momento, de una manera y con un contenido apropiados, de tal modo que permita (...) una evaluación pormenorizada del posible impacto $\mathrm{y}$, en su caso, preparar las consultas » con el fin de permitir que los representantes de los trabajadores expresasen " en un plazo razonable » una recomendación que "pueda ser tenida en cuenta en la empresa ». Dicho de otro modo, la consulta puede eventualmente desembocar en la firma de un acuerdo.

Para reforzar las posibilidades de intervención sindical en los CEE, las FSE crearon, después de la FEM, la figura del « coordinador» sindical para cada CEE. Dicho coordinador suele ser un funcionario sindical nacional, pero tiene una orden europea y debe por tanto velar por que el CEE actúe al servicio del interés general europeo de los asalariados de cada ETN.

\section{Nuevo impulso de la negociación transnacional: los acuerdos marco internacionales y europeos}

Tras impulsar una forma particular de negociación transnacional, consistente en la puesta en marcha de los CEE en la que las organizaciones sindicales no se ven implicadas más que parcialmente, la directiva sobre los CEE influyó enormemente en el desarrollo de la negociación colectiva de empresa transnacional, en un sentido próximo a los objetivos iniciales de las FSI. En efecto, cinco años después de la 
adopción de la directiva, los acuerdos transnacionales de empresa se multiplicaron tanto a nivel mundial como europeo.

Existe una fuerte correlación entre la adopción de la directiva sobre los CEE en 1994 y el impulso de los AMI y AME (da Costa y Rehfeldt 2012a). Antes de 1994, solo se firmaron cuatro AMI, todos para una sola empresa: BSN-Danone. El verdadero impulso cuantitativo de los AMI empieza en 2000, cuatro años después de la entrada en vigor de la directiva. Como hemos dicho, la mayoría de los ACI fue firmada por empresas del continente europeo, de las cuales todas, menos dos, habían instaurado previamente un CEE. 13 AMI (de los que 12 de los 26 firmados lo fueron por la FITIM) están o cofirmados por un CEE, o, en el caso de Volkswagen, Daimler Chrysler y Renault, por un comité de empresa mundial (CEM). Todos los AMI co-firmados por un CEE o un CEM, excepto uno, se negociaron con una empresa alemana o franco-alemana (EADS). A partir de 2006, la co-firma de los AM por parte de los CEE desaparece casi del todo, en paralelo a la disminución del número de AMI firmados por empresas alemanas.

La correlación es aún más fuerte en el caso de los AME. Los CEE firmaron la mayoría de los AME. Un único CEE firmó casi la mitad de los AME. Hasta 2013, tan solo 16 AME tuvieron unas FSE como únicas firmantes. Los demás AME fueron co-firmados por un conjunto de interlocutores por parte de los representantes de los asalariados, entre los que figuraban los sindicatos nacionales. La firma conjunta por un CEE y una o varias FSE es la figura más frecuente de esas combinaciones. Incluso cuando no firman, los CEE suelen estar implicados en la preparación de la negociación y/o en el proceso de seguimiento de los acuerdos.

El número de AME puede parecer escaso comparado con la totalidad de ETN en Europa. Sin embargo, la óptica cambia si se compara con el número de ETN que disponen de un CEE. En 2005, más de la cuarta parte (29\%) de las ETN francesas con CEE firmó al menos un AME. En el caso de las ETN alemanas, la tasa baja al $9 \%$ y, en el caso de las ETN norteamericanas o británicas al $6 \%$ y $2 \%$ respectivamente. Las ETN francesas firmantes de AME tienen una gran intensidad de negociación. Firmaron, por término medio, más de dos AME cada una. Únicamente las cuatro empresas norteamericanas firmantes de AME, que disponen todas de un CEE, tienen una mayor intensidad de negociación. En efecto, cada una firmó una media de cinco AME, General Motors Europe (ahora Opel-Vauxhall) firmó diez, Ford Europe cinco.

\section{La nueva estrategia de las federaciones sindicales europeas y sus límites}

Desde 2007, constatamos una nueva tendencia en la firma de los AME. Entre 1996 y 2006, los CEE firmaron, ellos solos, el 60\% de los AME; en cambio ahora, solo son los únicos firmantes del 39\% de los AME cerrados entre 2007 y 2011 . Este cambio de tendencia remite a un cambio de estrategia de las FSE en lo referente a la firma de acuerdos transnacionales. Así, tras apoyar sin reserva el desarrollo de los CEE y el ejercicio de sus derechos, las FSE empezaron a sentirse reticentes ante la 
evolución de las actividades de algunos CEE, desde la mera información-consulta hacia la negociación colectiva. Ese distanciamiento parte de una constatación y de un temor. La constatación consiste en el hecho de que los CEE distan mucho de estar, en la actualidad, mayoritariamente sindicalizados: únicamente los CEE de las empresas muy grandes lo están. De ahí su temor a que puedan negociar acuerdos que contravengan normas contractuales o legales a nivel nacional.

La FEM, a la que siguieron después otras FSE, adoptó en 2006 reglas internas que se proponían establecer un poder sindical y reservar la negociación y la firma de AME a las FSE (FEM, 2006). Se trata de un doble procedimiento de poder de un grupo de negociación y validación del acuerdo por parte de una mayoría de dos terceras partes al menos en las organizaciones sindicales de cada país implicado. El grupo de negociación puede contar también con miembros sindicados del CEE. Un equipo restringido bajo la dirección de la FEM, que firma sola el acuerdo tras su validación interna, lleva la negociación. La FEM decretó también la necesidad de incluir una cláusula de no regresión en cada acuerdo negociado ${ }^{10}$. Hasta 2011, la FEM consiguió negociar y firmar 8 AME según las nuevas reglas, exclusivamente con cinco ETN francesas (o antiguamente francesas): Areva, Schneider Electric, Arcelor Mittal, Thales, Alstom. La dirección de estas empresas era favorable al uso de dicho procedimiento por varios motivos. En primer lugar, está acostumbrada a negociar, con las organizaciones sindicales, acuerdos de empresa. Considera al CEE como un actor que se limita a un papel de información-consulta similar al de un comité de empresa francés. Ya hemos mencionado que algunos DRH desean extender la cultura de diálogo social de su grupo a las filiales extranjeras. Asimismo, puede resultar interesante negociar directamente, a nivel europeo, sobre temas en los que debe negociar obligatoriamente, debido a la legislación francesa, un acuerdo a nivel del grupo francés. La negociación con la FEM tiene la ventaja de no verse confrontada más que con un único interlocutor y no con una multitud de organizaciones sindicales, como es el caso de una negociación a nivel de un grupo francés. La carga de la coordinación intersindical le corresponde así a los sindicatos franceses y a la FEM.

Las organizaciones sindicales francesas aceptaron desempeñar el papel de la coordinación europea y confiar la orden de negociación a la FEM; al igual que la

${ }^{10}$ Hasta la fecha, ningún AME ha cuestionado una regla convencional nacional o europea: no suelen sino aportar algo más a los derechos existentes. Algunos acuerdos de reestructuración, en especial en General Motors Europe, constituyen sin embargo casos límites. En efecto, estos acuerdos intercambian a veces concesiones a nivel de la organización del trabajo por garantías para el empleo y la salvaguarda de las plantas. En este caso concreto, dichas concesiones mutuas fueron sometidas a votación y puestas en marcha mediante acuerdos locales de aplicación del acuerdo marco europeo (da Costa et Rehfeldt 2009). 
dirección, prefieren una negociación con una FSE a una negociación con el CEE. ${ }^{11}$ En efecto, desde hace mucho tiempo, algunas organizaciones francesas se temen que los CEE se extralimiten en su papel de información-consulta. De ello resulta su tradicional elección consistente en no designar figuras muy centrales de las relaciones laborales como representantes en el CEE y, a fortiori, como su secretario. Finalmente, el sistema francés de relaciones laborales, que abarca una jerarquía de las normas mediante el principio de favor, facilita la delegación de la orden para negociar a nivel transnacional, sin temor a que el AME pueda eventualmente contravenir in pejus las reglas vigentes. Por todos estos motivos, que no son exhaustivos, las empresas francesas tienen una propensión muy superior a sus homólogas alemanas no solo a firmar unos AME sino también a aplicar los nuevos procedimientos de las FSE - siempre y cuando lo soliciten las organizaciones sindicales presentes en el CEE. En efecto, aunque las ETN francesas aceptan con mayor facilidad que las de otros países la firma de unos AME según los nuevos procedimientos de las FSE, algunas siguen negociándolos con los CEE solos y/o sindicatos nacionales con o sin la participación de las FSE.

Sin embargo, la nueva estrategia sindical europea para la negociación colectiva transnacional de empresa se enfrenta con la práctica de los CEE en las ETN alemanas. Ahora bien, éstas están en mayor número entre las grandes ETN europeas. De las 1642 ETN que dependían teóricamente de la directiva CEE en 2005, 450 tenían su sede en Alemania, 344 en Estados Unidos, 265 en el Reino Unido y 210 en Francia. Pese a la escasa tasa de conformidad - claramente menor que la de las ETN francesas, británicas y norteamericanas, que se sitúan por encima de la media - las empresas alemanas tenían en 2005 el mayor número (123) de las 772 empresas que disponían por aquellas fechas de un CEE, frente a las empresas norteamericanas (120), británicas (109) y francesas (79) (Kerckhofs, 2006; 29).

En consecuencia, las FSE pueden difícilmente eludir la realidad de las ETN alemanas. Su impacto sobre la negociación transnacional es tanto mayor cuanto que la mayoría de los temas que son, en la actualidad, objeto de AME, son precisamente temas que dependen en Alemania de la competencia, aunque no sea exclusiva, de los consejos de empresa. En efecto, en Alemania, los consejos de empresa tienen derecho a negociar acuerdos de empresa sobre todos los temas que no suelen depender de la negociación colectiva de rama (especialmente los temas de salarios y tiempo de trabajo). Ahora bien, en la mayoría de los CEE de las ETN alemanas, la óptica de los representantes de los asalariados alemanes influye, en distintos grados, en las prácticas de dichos CEE. El canal principal de dicha influencia pasa por el

\footnotetext{
${ }^{11}$ Es más, de modo inesperado, eso incitaba a la Federación Francesa de Cuadros de la Metalurgia a afiliarse a la FEM para no verse excluida de estas negociaciones centralizadas. Esta federación está afiliada, a nivel nacional, a la CFE-CGC, que a su vez está afiliada, a nivel europeo, a la Confederación Europea de Cuadros (CEC) y no a la CES.
} 
presidente del CEE, que suele ser un asalariado alemán y que junta esta función con la presidente del consejo central de empresa o del consejo de grupo de la sede alemana. Este tiene tendencia, conscientemente o no, a trasladar las prácticas alemanas al plano europeo.

Las organizaciones sindicales alemanas suelen velar por que los consejos de empresa no se extralimiten en sus competencias legales, aunque resulte a veces difícil frente a presidentes de consejos de empresa fuertemente apoyados por los asalariados que los han elegido. Semejante control resulta más difícil sobre las prácticas de los CEE. Incluso una organización tan potente como IG Metall, suele ser incapaz de desaprobar una práctica de negociación de AME por el CEE solo, e imponer el respeto de las reglas de negociación adoptadas por la FEM (ver el análisis de algunos de esos casos controvertidos por Müller et al. 2011 y 2013). Las preferencias de procedimiento de algunos presidentes alemanes de CEE se ven contrariados en muy pocos casos por una dirección de las ETN alemanas que no suele desear desestabilizar el diálogo social en el plano nacional por un conflicto relativo al procedimiento a nivel europeo. Dicha configuración de intereses y prácticas puede explicar la preferencia de las empresas alemanas en dar un papel de líder al CEE en la negociación de un AME. Puede explicar también por qué la firma de AME por las ETN alemanas disminuyó considerablemente desde 2009. Sin embargo, es muy pronto aún para llegar a la conclusión de que se trata de una evolución irreversible.

\section{Conclusión: ¿Cuáles son las perspectivas para dar un nuevo impulso a los AME?}

La evolución cuantitativa de los acuerdos transnacionales firmados en 20122013 muestra una continuación de la dinámica pasada de los AMI, aunque también cierto debilitamiento de la dinámica de los AME, debido entre otras cosas a un menor número de AME firmados por empresas alemanas. Las ETN francesas son las que, en la actualidad, llevan casi exclusivamente el peso de los AME; firmaron 10 de los 13 AME firmados en 2012-2013, unas empresas alemanas firmaron los tres restantes. Podemos preguntarnos si eso supone un debilitamiento pasajero debido a la crisis económica actual. En efecto, la crisis ha provocado en todos los países un nuevo centrado nacional de las relaciones larorales. En los años 20002010, algunos AME, entre otros en el sector del automóvil, intentaron encontrar soluciones europeas a problemas de reestructuración transnacional (da Costa y Rehfeldt 2009 y 2012b), muy pocos AME abordan la cuestión en el período reciente. La empresa pionera General Motors Europe firmó nueve AME sobre reestructuraciones entre 2000 y 2010 que garantizaban el empleo del conjunto de sus empresas, pero desde aquella fecha, no firmó ninguno más. Pese a todo, no por ello pararon las reestructuraciones; pero son objeto ahora de acuerdos negociados únicamente en el plano nacional, tal y como indica el ejemplo del acuerdo alemán de 2012 sobre el cierre de la fábrica GM de Bochum. De igual manera, Ford Europe, 
que firmó cinco acuerdos de reestructuraciones entre 2000 y 2006, no firmó ninguno más desde la fecha, aunque nuevas reestructuraciones y nuevos cierres están previstos. Esta nueva nacionalización de las relaciones laborales se debe seguramente a un creciente intervencionismo de los Estados nacionales en la crisis. Sobre todo en la industria automóvil, algunos gobiernos apoyaron desde el punto de vista financiero su industria automóvil incitándola a renunciar temporalmente a despidos colectivos (Pedersini 2010).

En lo referente a las partes firmantes de los AME, vemos en 2012-2013 la misma variedad de prácticas que en el pasado. Aunque tres de los diez AME franceses de aquel período son firmados por una FSE sola (y por tanto respetan las consignas del modelo de la FEM de 2006), los o bien son firmados conjuntamente con un CEE, bien firmados por un CEE solo. Esta continuidad en la variedad hace necesaria una clarificación del debate sindical a nivel europeo, y debería hacerse también tanto en el plano de la CES como de las FSE.

En la última década, estamos observando una cohabitación, en la antigua FEM, de varias líneas estratégicas. Para esquematizar, podríamos calificar una de ellas de "pragmática". Se encontraba más cercana a los CEE y se hallaba en el comité de la política de la empresa de la FEM, comité compuesto por expertos de las distintas organizaciones miembros en materia de CEE y que se encargaba sobre todo de las reestructuaciones. Este comité elaboró un documento sobre « el enfoque político de la FEM en materia de reestructuraciones de empresa socialmente responsable » que fue adoptado por el Comité Ejecutivo de la FEM en junio de 2005 y publicado en un manual específico (FEM 2005). Este documento preveía el establecimiento de un dispositivo de alerta que le concede un papel central al coordinador FEM del CEE, lo cual satisface bastante a los sindicalistas alemanes. En caso de anuncio de una reestructuración transnacional, la FEM debía poner en marcha un "grupo de coordinación sindical », compuesto por representantes de los sindicatos implicados en la empresa que se tenía que reestructurar, por el CEE y por el coordinador FEM. Este grupo de coordinación sindical estaba estructurado sobre el modelo de la negociación exitosa de un AME sobre la reestructuración de General Motors Europe en 2004. En caso necesario, podía transformarse en grupo de negociación de un acuerdo marco que fijaba principios de garantía del empleo ante cualquier negociación en el plano nacional.

Otra línea sindical en la FEM defendía una política más autónoma frente a los CEE. Se encontraba en el comité de negociación colectiva de la FEM, comité compuesto por funcionarios sindicales nacionales encargados de esta materia. Este comité había elaborado las reglas internas de 2006 presentadas anteriormente que endurecían el procedimiento de negociación de 2005. Este documento tiene mayor influencia que la postura sindical italiana, que exigía que la negociación con una ETN fuese llevada bajo la égida de la FEM sola y que la FEM sola firmase los acuerdos (Dufresne 2012). Ya no menciona un « grupo de coordinación sindical », sino tan solo un «equipo de negociadores" compuesto obligatoriamente por representantes de la FEM y sindicatos nacionales. Ya no se busca de modo explícito 
la participación de representantes del CEE, sino que solo se invoca como posibilidad.

Algunas señales indican que las posturas de unos y otros han evolucionado para dar paso a soluciones de compromiso. Ya en el pasado, el grupo franco-alemán EADS había negociado y firmado, en 2010, un acuerdo de procedimiento para la negociación de futuros AME que no se ceñía exactamente a las reglas internas de la FEM de 2006, aunque respetaba uno de sus principios, aquel según el cual son los sindicalistas los que deben negociar esos AME, con un poder de sus respectivas organizaciones nacionales. ${ }^{12} \mathrm{El}$ acuerdo firmado por esta empresa permite combinar las particularidades del sistema alemán con el predominio sindical reinante en las otras filiales extranjeras. La FEM, que no había participado en la negociación del acuerdo de procedimiento, tendría sin embargo un lugar en la negociación de los futuros AME en EADS. Para el antiguo secretario general de la FEM, que era también el coordinador sindical del CEE de EADS, el acuerdo suponía « un paso en la buena dirección », aunque el procedimiento no se aplica integralmente.

Recientemente, observamos también una evolución hacia un enfoque estratégico más pragmático en la CES y sus federaciones. En una « nota de discusión » de junio 2012, el Comité Ejecutivo de la CES ha procurado armonizar las posturas de las distintas FSE en materia de negociación de empresa transnacional (ETUC 2012). De ahí se desprende que la postura inicial de la FEM de 2006 no es ahora tan preponderante desde que se fusionó con otras dos FSE para crear la nueva FSE « IndustriAll Europe ». Las nuevas reglas de IndustriAll Europe conceden un papel más importante al CEE, consistente en crear un « entorno propicio a la negociación transnacional ». El CEE debe por tanto estar implicado desde el principio en el proceso de negociación. Los miembros sindicalizados del CEE son considerados ahora como una componente regular del grupo de negociación. Sin embargo, su organización sindical debe darles el poder, al mismo título que los permanentes sindicales miembros del grupo. En su conclusión, la nota subraya la fuerte proximidad de las reglas de las diferentes FSE. Sin embargo, insiste en la necesidad de incluir a miembros sindicados del CEE en el grupo de negociación.

${ }^{12}$ El acuerdo de procedimiento EADS fue negociado y firmado exclusivamente por sindicalistas designados por sus respectivas organizaciones de Francia, Alemania, España y Reino Unido. Desde ahora en adelante, los AME del grupo EADS serán negociados por un «grupo europeo de negociación » compuesto, a prorrata del número de asalariados representados, por negociadores designados por sus organizaciones sindicales (en Alemania por el consejo de empresa del grupo), y también por los dos presidentes (asalariados) del CEE designados por este. Un representante de la FEM participará como « coordinador y consejero ». Para que el acuerdo se pueda aplicar en un país dado, debe ser validado por, al menos, las dos terceras partes de los representantes de los miembros del grupo de negociación. 
Queda por ver ahora si este nuevo equilibrio de procedimiento entre FSE y CEE, propuesto por la CES, podrá reducir las divergencias de prácticas observadas en el pasado y dar una nueva dinámica a la negociación transnacional. Dado que las FSE no tienen, en la actualidad, ningún medio legal para obligar a sus afiliados $\mathrm{y}$, a fortiori, a los CEE o las ETN a respetar sus reglas internas, la vía de un acercamiento libremente consentido por todos los actores parece ser la única forma de conseguir una homogeneización de los procedimientos que no produzca un debilitamiento de la negociación colectiva transnacional.

\section{Bibliografía}

Commission européenne (2013). Base de données sur les accords d'entreprise transnationaux, http://ec.europa.eu/social/main.jsp?catId=978\&langId=fr

da Costa I., Rehfeldt U. (2008). Convenios colectivos trasnacionales en el ámbito de la empresa: avances históricos, in: Papadakis, K. et al., Diálogo social y acuerdos transfronterizos. ¿Un marco global emergente de relaciones industriales?, Ministerio de Trabajo e Inmigración: 2009, (Colección Informes OIT, núm. 82), pp. 69-94.

da Costa I., Rehfeldt U. (2009). Les CEE et la négociation collective transnationales : les accords européens et mondiaux dans l'automobile. La Revue de l'IRES, $\mathrm{n}^{\circ}$ 61, pp. 99-127.

da Costa I., Rehfeldt U. (2012a). Les négociations collectives transnationales: dynamiques des accords-cadres européens et mondiaux. La Revue de l'IRES, $\mathrm{n}^{\circ} 71$, pp. 115-146.

da Costa I., Rehfeldt U. (2012b). Transnational Company Agreements on restructuring at EU level, in: Leonardi S. (ed.), Transnational Company Agreements: a stepping stone towards a real internationalization of industrial relations, Rome: Ediesse; pp. 85-104.

da Costa I., Rehfeldt U., Müller T., Telljohann V. y Zimmer R. (2010). Les accordscadres européens et internationaux: nouveaux outils pour des relations professionnelles transnationales . La Revue de l'IRES N 66, pp. 93-116.

Degryse C. et al. (2011). Dialogue social européen: bilan et perspectives, Bruselas: CES/OSE.

Dufresne A. (2011). Le salaire, un enjeu pour l'euro-syndicalisme. Histoire de la coordination des négociations collectives nationales, Nancy: PUN. 
Dufresne, A. (2012). Trade union support and political blocage: the actors' viewpoint. European Journal of Industrial Relations, 18 (2) ; pp. 107-121.

ETUC (2012). More and Better European Company Framework Agreements: Enhancing Trade Unions in Transnational Negotiations with Transnational Companies (Discussion Note), Executive Committee, Bruselas, 5-6 Junio.

European Commission (2012). Social Europe Guide, Vol. 2: Social Dialogue, Bruselas, Enero.

FEM (2005). Política de la FEM enfocada a la reestructuración socialmente responsable de las empresas. Aprobada por el $100^{\circ}$ Comité Ejecutivo de la FEM, Luxemburgo, 7 y 8 de junio de 2005, en: Como gestionar las reestructuraciones transfronterizas de las empresas. Versión española del manual de la FEM. Bruselas: Federación Europea de Metalúrgicos, pp. 11-15.

FEM (2006). Procédure interne de la FEM pour les négociations au niveau des entreprises multinationales adoptée par le $102^{\mathrm{e}}$ Comité exécutif de la FEM, 1314 juin, in: Jalons de Rome à Madrid, Supplément 2005-2009, Bruselas: Fédération Européenne des Métallurgistes, 2010, Annexe 1, p. 13-15.

Fundación $1^{\circ}$ de Mayo. Los Acuerdos Transnacionales de Empresa en la práctica: fortalezas y debilidades. Seminario internacional, 6 Mayo 2014. Boletin digital $\mathrm{N}^{\circ} \quad 36, \quad$ Mayo 2014. www. 1 mayo.ccoo.es/nova/NNws_ShwNewDup?codigo $=4551 \&$ cod_primaria $=1$ 194\&cod_secundaria $=1194 \# . U 3 \mathrm{kY}$ aXYRFkg

Gallin D. (2008). International framework agreements: A reassessment in Papadakis K. (ed.), Cross-Border Social Dialogue and Agreements: An Emerging Global Industrial Relations Framework?, Ginebra: ILLS/ILO, pp. 15-41.

Gonzalez Begega S. (2011). Empresa transnacional y nuevas relaciones laborales. La experiencia de los comités de empresa europeos, Madrid: Catarata.

Jagodziński R. (2012). Les Comités d'entreprise européens 18 ans après la directive: une evaluation. La Revue de l'IRES, n 71, pp. 51-70.

Kerckhofs P. (2006). European Works Councils. Facts and Figures 2006, Bruselas: ETUI-REHS.

Köhler H.-D., Gonzalez Begega S., (2007). Still learning from Europe. Spanish participation in European Works Councils, in: Whittal M., Knudsen H., Huijgen F. (eds.) Towards a European Labour Identity. The case of the European Works Council, Londres/Nueva York: Routledge ; 132-149. 
Levinson, C. 1972. Le contre-pouvoir multinational, París: Seuil.

Moore, H. (1978). Dunlop-Pirelli: Internationales Shop Steward-Komitee versus Weltkonzernausschuss, in: Olle, W. (ed.), Einführung in die internationale Gewerkschaftspolitik, t. 1, Berlín: Olle \& Wolter; pp. 97-106.

Müller T., Platzer H.W., Rüb S. (2011). European Collective Agreements at Company Level and the Relationship between EWCs and Trade Unions Lessons from the Metal Sector. Transfer, vol. 17, $\mathrm{n}^{\circ}$ 2, pp. 217-228.

Müller T., Platzer H.W., Rüb S. (2013). Transnational company agreements and the role of European Works Councils in negotiations. ETUI Report 121, Bruselas, 2013

Pedersini R. (2011). Social dialogue and recession in the automotive sector. Luxembourg: Eurofound/Publication Office of the European Union, 2010.

Piehl, E. (1974). Multinationale Konzerne und internationale Gewerkschaftsbewegung, Francfort/Main : EVA, 1974.

Philip, S. (1978). Weltkonzernausschüsse und Spaltung der internationalen Gewerkschaftsbewegung - das Beispiel Michelin. in : Olle, W. (ed.), Einführung in die internationale Gewerkschaftspolitik, t. 1, Berlin : Olle \& Wolter; pp. 79-96.

Pochet P. (2005). Le dialogue social sectoriel, une analyse quantitative. Chronique internationale de l'IRES $\mathrm{N}^{\circ} 96$, septiembre.

Pochet P. (2006). Le dialogue social interprofessionnel, une analyse quantitative. Chronique internationale de l'IRES $\mathrm{N}^{\circ} 98$, enero.

Rehfeldt U. (1993). Les syndicats européens face à la transnationalisation des entreprises. Le Mouvement social $\mathrm{N}^{\circ} 162$, enero-marzo, pp.69-93.

Rehfeldt U. (1998a). Die Renault-Vilvoorde-Affäre und ihre Bedeutung für die europäische Gewerkschaftspolitik. WSI-Mitteilungen No. 7, pp. 450-459.

Rehfeldt U. (1998b). European Works Councils - An Assessment of French Initiatives. in: Lecher W., Platzer H.W. (eds.), European Union - European Industrial Relations?, Londres/Nueva York: Routledge, pp. 207-222.

Rehfeldt U. (2009). La participation des salariés dans la Société européenne, in: Conchon A., Auberger M.-N. (dir.), Les administrateurs salariés et la gouvernance d'entreprise, París: La Documentation française, pp. 45-55. 
Tudyka K. (1986). Die Weltkonzernräte in der Krise. WSI-Mitteilungen, No. 4.

Waddington J. (2012). Comités d'entreprise européens : comment les salariés peuvent-ils exercer une influence ?. La Revue de l'IRES, n ${ }^{\circ}$ 11, pp.23-50.

White R., Vallejo C. (2004). Developments in European Works Councils - case of Spain. EIRO Online, www.eurofound.europa.eu/eiro/2004/11/study/index.htm 